\title{
Will an Asymptomatic Meningioma Grow or Not Grow? A Meta-analysis
}

\author{
Lingcheng Zeng ${ }^{1}$ Pei Liang ${ }^{2}$ Jiantong Jiao ${ }^{1}$ Jian Chen ${ }^{1}$ Ting Lei ${ }^{1}$ \\ ${ }^{1}$ Department of Neurosurgery, Tongji Hospital, Tongji Medical \\ College, Huazhong University of Science and Technology, \\ Hubei, China \\ 2 Sensory Science Laboratory, School of Bioscience and Food \\ Engineering, Changshu Institute of Technology, China

\begin{abstract}
Address for correspondence Jian Chen, MD, Department of Neurosurgery, Tongji Hospital, Tongji Medical College, Huazhong University of Science and Technology, Jiefang Dadao No.1095, Wuhan,
\end{abstract} \\ Hubei 430030, China (e-mail: tjcj39280@163.com).
}

J Neurol Surg A 2015;76:341-347.

\begin{abstract}
Keywords

- asymptomatic meningioma

- natural history

- meta-analysis
\end{abstract}

\section{Introduction}

Meningioma is one of the most common benign types of intracranial tumors. It grows from arachnoid brain cells and clinically makes up $13-26 \%$ of all primary intracranial tumors. ${ }^{1}$ Due to the compression of the tumor to adjacent vessels and nerves, various clinical symptoms may develop. ${ }^{1}$ Depending on the location and the size of the tumor, surgery and stereotactic radiotherapy are the common treatments to

received

May 6, 2014

accepted after revision

October 24, 2014

published online

March 23, 2015

control tumor growth, relieve symptoms, and improve quality of life. $^{1}$

In recent years, with the extensive application of head computed tomography (CT) and magnetic resonance imaging (MRI) examination, more asymptomatic meningiomas have been detected incidentally. ${ }^{2}$ The patients manifest no tumorrelated symptoms or only show nonspecific symptoms such as mild headache and dizziness. ${ }^{3}$ The decision to operate on such patients should be based on careful weighing of the

(c) 2015 Georg Thieme Verlag KG Stuttgart · New York
DOI http://dx.doi.org/ $10.1055 / \mathrm{s}-0034-1543959$. ISSN 2193-6315. 
surgical risks and benefits. At the early stage, the benefits of surgery are to achieve a lower Simpson grade resection and reduce operative risks associated with a relatively smaller tumor size compared with those at a later stage with obvious symptoms. ${ }^{4}$ Now an important question arises: How does asymptomatic meningioma progress, or when will the symptoms develop?

Previous studies on the growth pattern of meningioma demonstrated that most meningiomas grew indolently or even no growth is observed for many years. ${ }^{2,5-7}$ In the early 1990s, Firsching et al $^{5}$ studied the growth rate of asymptomatic meningioma by CT follow-up of 17 patients. In the followup period of $\sim 21$ months, no growth or only minor growth was observed in most cases, and none of those patients underwent surgery. ${ }^{5}$ Another follow-up study of 67 patients with asymptomatic meningioma was analyzed for at least 5 years. It showed that 42 of 67 cases $(\sim 62.7 \%)$ manifested no obvious growth. ${ }^{4}$ Braunstein and Vick $^{6}$ even followed one parietal falx meningioma and one frontal convexity meningioma for up to 12.8 years, and still no growth of tumors was observed. An epidemiological study conducted in Germany demonstrated that from 1961 to 1986 , there was an annual incident detection of meningioma of 1.85 per 100,000 population. Interestingly, up to half of the proportion was discovered by autopsies, suggesting that some patients did not even know they had a meningioma all their lives. ${ }^{7}$ Therefore, asymptomatic meningioma may remain undiscovered because of the tumor's slow growth. In such cases, conservative follow-up is apparently a better choice than an operation because it avoids postsurgical complications.

The key to a conservative treatment decision is the careful evaluation of the growth possibilities of the meningioma that can be predicted using a patient's clinicoradiologic factors such as gender, tumor location, calcification, MRI T2 signal intensity, and peritumoral brain edema. However, so far the associations between tumor growth and the parameters just listed have not always been consistent. This study aimed to further clarify the meningioma growth-related factors with a meta-analysis. We compared our results with previous reports; consistencies and the inconsistencies are discussed.

\section{Materials and Methods}

\section{Literature Search}

The literature was searched via PubMed, Embase, and the ISI Web of Knowledge from January 1970 to August 2013. The following keywords were chosen for the electronic search: asymptomatic meningioma, incidental meningioma, small meningioma, natural history, growth, conservative treatment, conservative therapy, and follow-up. To cover the maximum number of eligible studies, different combinations of the keywords were applied to the literature search. The references of all eligible articles were searched again, in case they were not covered by the electronic searches the first time.

\section{Selection Criteria}

The following criteria were used to select the data for the meta-analysis: (1) The cohort or case-control original studies presented quantitative data on the association between clinical or radiologic factors and growth of meningioma; (2) tumor growth was demonstrated on serial MRI or CT imaging; (3) the language of the publications should be confined to English; (4) the studies would be excluded if the full text could not be found or if the published data were insufficient to estimate an odds ratio (OR) and a confidence interval (CI); and (5) when there were multiple articles by the same group based on similar patients, only the most recent article was included.

\section{Data Extraction}

Two independent investigators extracted all relevant data from the included studies including author, year, country, patient number, mean follow-up duration, growth measurements, cutoff score for tumor growth, and factors related to tumor growth. Disagreements about any data extraction by the two investigators were referred back to the original article to achieve consensus.

\section{Statistical Analysis}

Review Manager v. 5.2 was applied for data analysis. Comparisons of dichotomous measurements were performed with the pooled estimates of ORs, as well as the $95 \%$ CIs. A $p$ value $<0.05$ was considered statistically significant. Between-study heterogeneity was evaluated using the $\mathrm{I}^{2}$ statistic. If $\mathrm{I}^{2}>50 \%$, it indicates statistically significant heterogeneity. If there was heterogeneity, the random model was adopted; otherwise the fixed model was used. Sensitivity analysis was performed to evaluate the stability of the pooled estimates by exclusion of specific studies. Publication bias was assessed by the Begg rank correlation and the Egger weighted regression. A $p$ value $<0.05$ was considered a statistically significant publication bias.

\section{Results}

\section{Search Results and Study Characteristics}

A total of 1,120 duplicates among the three databases and 203 non-English articles were removed from the initial 3,315 records, leaving 1,992 articles to review. According to the titles and abstracts, 33 of them were potentially eligible and the full texts were reviewed. Overall, 24 articles were excluded and 9 articles were included according to the selection criteria outlined earlier. - Fig. 1 shows a flowchart of the selection process. - Table 1 lists the details of the included studies.

\section{Association between Tumor Growth and Clinicoradiologic Factors}

The association between tumor growth and clinicoradiologic parameters is analyzed and illustrated in - Fig. 2. It shows that tumor growth was negatively correlated with tumor calcification (pooled OR: 0.23; 95\% CI, 0.11-0.46; $p<0.001$; random effect; - Fig. 2A) but positively associated with MRI T2 signal intensity, leading to a risk difference of 2.75 (95\% CI, 1.75-4.33; $p<0.001$; fixed effect; - Fig. 2B). No association was found between tumor growth and other features such as sex (pooled OR: 1.29; 95\% CI, 0.84-1.99; 


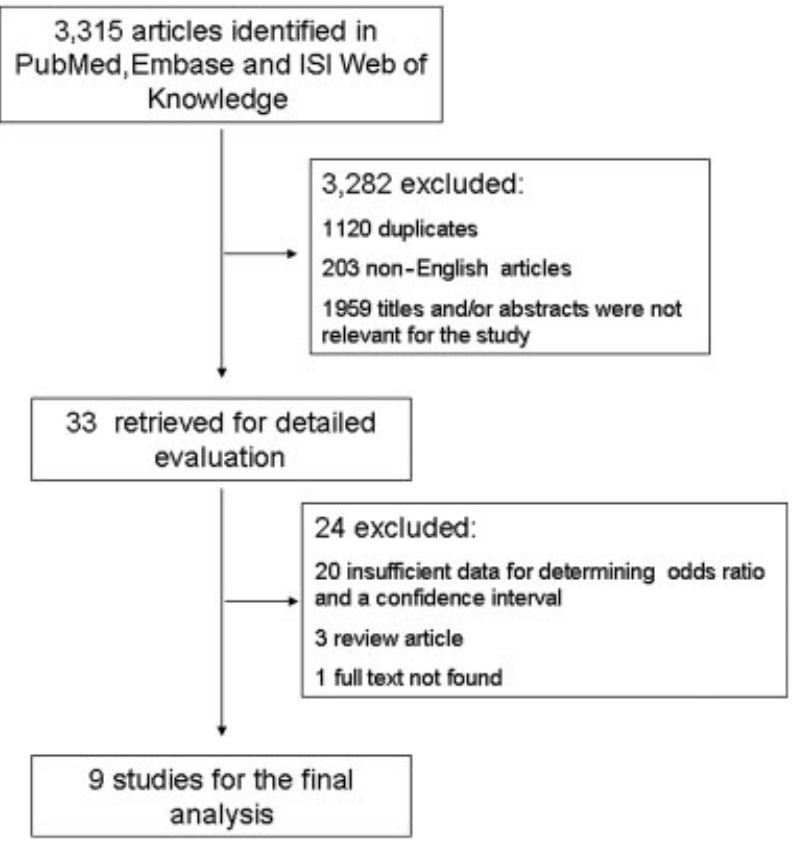

Fig. 1 Flowchart for selection of studies.

$p=0.24$; fixed effect; - Fig. $2 C$ ), skull base location (pooled OR: $\quad 0.80 ; \quad 95 \% \quad \mathrm{Cl}, \quad 0.25-2.58 ; \quad p=0.70 ; \quad$ random effect; - Fig. 2D), and peritumoral brain edema (pooled OR: 1.24; 95\% CI, 0.29-5.27; $p=0.77$; random effect; - Fig. 2E).

\section{Subgroup and Sensitivity Analysis}

To examine whether the pooled data of the growth-related factors were influenced by different growth measurements on serial imaging and to evaluate the potential source of heterogeneity, the subgroups of tumor volumetry and maximum diameter measurement were further classified, and the subgroup analysis was performed accordingly (-Table 2 ). Despite different methods of measurements, calcification and MRI T2 hyposignal still indicated a significantly slow growth of meningioma. Other factors such as sex and peritumoral brain edema nevertheless had no significant correlation with tumor growth. Only one previous study ${ }^{8}$ adopting the method of volumetric analysis suggested a negative association between skull base location and tumor growth. The other three studies ${ }^{4,9,10}$ using maximum diameter measurement found no correlation between them.

In addition, exclusion of one study at a time had no influence on the main effect. For instance, regarding the factor of tumor calcification, if the studies of Rubin et al and Oya et al were excluded from the group, the $\mathrm{I}^{2}$ value decreased from $55.2 \%$ to $0.0 \%$. However, the pooled OR is 0.24 (95\% CI, 0.13-0.44; $p<0.001$, fixed effect) and still indicates a significant association between calcification and slow tumor growth. Similarly, for the factor of MRI T2 signal intensity, random omission of any one study did not influence the positive correlation between MRI T2 signal intensity and tumor growth ( $p<0.001$; fixed effect).

\section{Publication Bias}

The $p$ values of the Begg test varied from 0.22 to 1.00; those of the Egger test varied from 0.23 to 0.94 for all the previously mentioned meta-analyses. Because in both the bias estimation models the $p$ values were $>0.05$ and the total number of studies for each factor was limited $(<10)$, no funnel plot was applied.

\section{Discussion}

The clinical and radiologic characteristics of patients are important to identify the growth status of a meningioma and predict its development. Many articles have studied factors relating to tumor growth. However, many of their observations are inconsistent. With our meta-analysis we studied a total of 777 samples of meningiomas and analyzed the correlation between tumor growth potentials and clinical factors including gender, tumor location, calcification, MRI T2 signal intensity, and peritumoral brain edema.

The female hormones progesterone and estrogen play a role in the development of meningioma and are important for the

Table 1 Characteristics of included studies

\begin{tabular}{|c|c|c|c|c|c|c|}
\hline Study & Year & Country & No. of patients & Mean FU, mo & Growth measurement & $\begin{array}{l}\text { Cutoff score for } \\
\text { growth definition }\end{array}$ \\
\hline Yoneoka et al ${ }^{26}$ & 2000 & Japan & 37 & 50.4 & Volumetry & $1 \mathrm{~cm}^{3} / \mathrm{y}$ \\
\hline Kuratsu et al ${ }^{19}$ & 2000 & Japan & 63 & 36.6 & Volumetry & NS \\
\hline Hashiba et al $^{15}$ & 2009 & Japan & 70 & 39.3 & Volumetry & $15 \%$ \\
\hline Hashimoto et al ${ }^{8}$ & 2012 & Japan & 113 & 46.9 & Volumetry & NS \\
\hline Nirro et al $^{18}$ & 2000 & Japan & 40 & 38.4 & Maximum diameter & NS \\
\hline Herscovici et al ${ }^{30}$ & 2004 & Israel & 51 & 67 & Maximum diameter & $2 \mathrm{~mm}$ \\
\hline Yano and Kuratsu ${ }^{4}$ & 2006 & Japan & 67 & $>60$ & Maximum diameter & NS \\
\hline Rubin et $\mathrm{al}^{9}$ & 2011 & Israel & 63 & 65 & Maximum diameter & NS \\
\hline Oya et al ${ }^{10}$ & 2011 & USA & 273 & 45.6 & Maximum diameter & $2 \mathrm{~mm}$ \\
\hline
\end{tabular}

Abbreviations: FU, follow-up; NS, not specified. 


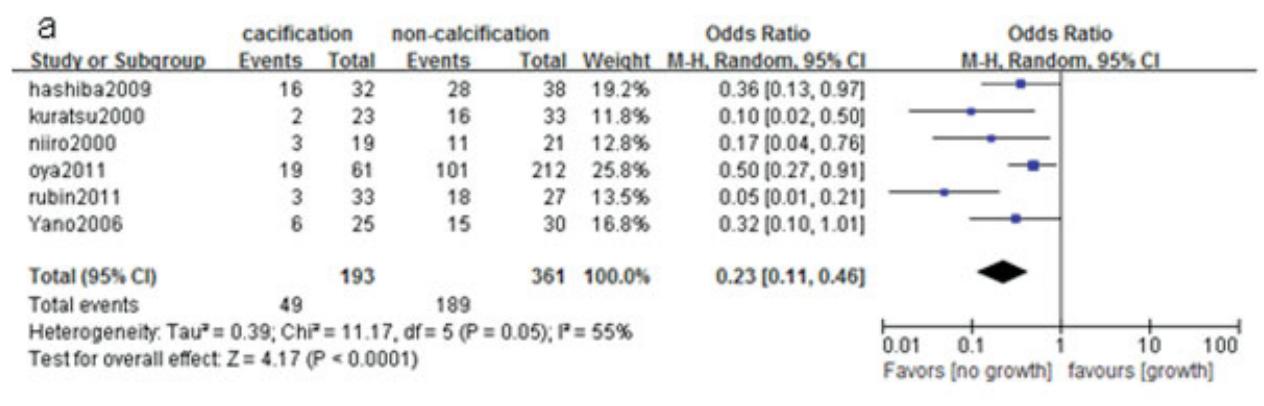

\begin{tabular}{|c|c|c|c|c|c|c|c|c|c|}
\hline \multirow{2}{*}{$\begin{array}{l}\mathrm{b} \\
\text { Study or Subqroup }\end{array}$} & \multicolumn{2}{|c|}{ Hyper signal } & \multicolumn{2}{|c|}{ Hypo signal } & \multirow[b]{2}{*}{ Weight } & \multirow{2}{*}{$\begin{array}{c}\text { Odds Ratio } \\
\text { M- }-\mathrm{H}, \text { Fixed, } 95 \% \mathrm{Cl}\end{array}$} & \multirow{2}{*}{\multicolumn{2}{|c|}{$\begin{array}{c}\text { Odds Ratio } \\
\text { M.H. Fixed, } 95 \mathrm{~s} \text { Cl }\end{array}$}} & \\
\hline & Events & Total & Events & Total & & & & & \\
\hline hashiba2009 & 24 & 36 & 20 & 34 & $30.0 \%$ & $1.40[0.53,3.70]$ & & & \\
\hline kuratsu2000 & 8 & 14 & 1 & 19 & $1.6 \%$ & $24.00[2.47,233.45]$ & & & \\
\hline niiro2000 & 7 & 10 & 7 & 17 & $6.8 \%$ & $3.33[0.63,17.57]$ & & & \\
\hline oya2011 & 32 & 49 & 86 & 214 & $48.7 \%$ & $2.80[1.46,5.36]$ & & - & \\
\hline Yano2006 & 11 & 20 & 7 & 23 & $12.8 \%$ & $2.79[0.80,9.76]$ & & & \\
\hline Total $(95 \% \mathrm{Cl})$ & & 129 & & 307 & $100.0 \%$ & $2.75[1.75,4.33]$ & & & \\
\hline Total events & 82 & & 121 & & & & & & \\
\hline $\begin{array}{l}\text { Heterogeneity. } \mathrm{Chi}^{2} \\
\text { Test for overall effec }\end{array}$ & $\begin{array}{l}5.39, d f= \\
Z=4.38\end{array}$ & $\begin{array}{l}(P=0 \\
<0.00\end{array}$ & $\begin{array}{l}\text { 25): } P=2 \\
\text { 01) }\end{array}$ & $26 \%$ & & & $\begin{array}{ll}0.01 & 0.1 \\
\text { Favors ino }\end{array}$ & $\begin{array}{r}10 \\
\text { favours } 10 \mathrm{r}\end{array}$ & 100 \\
\hline
\end{tabular}

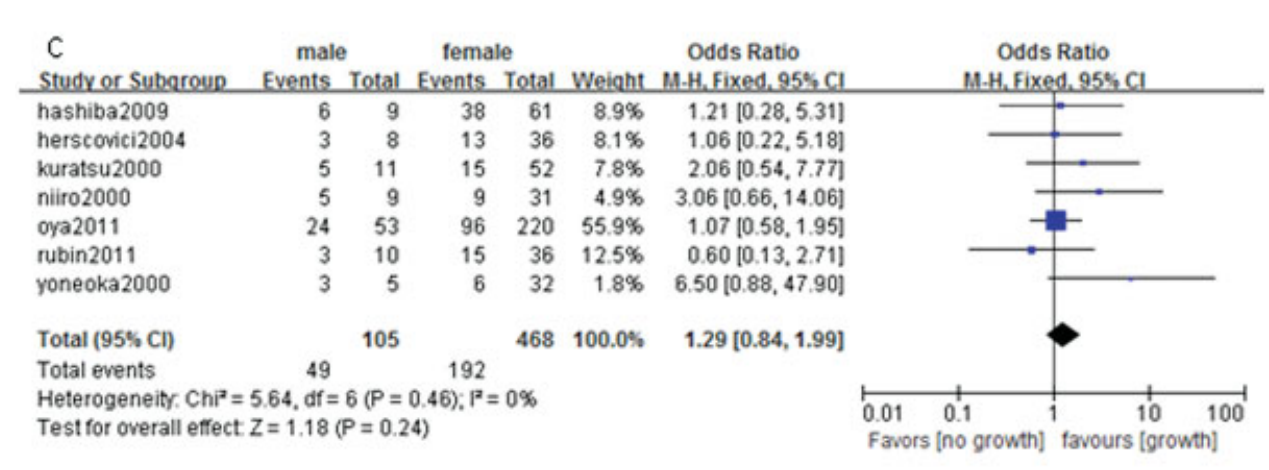
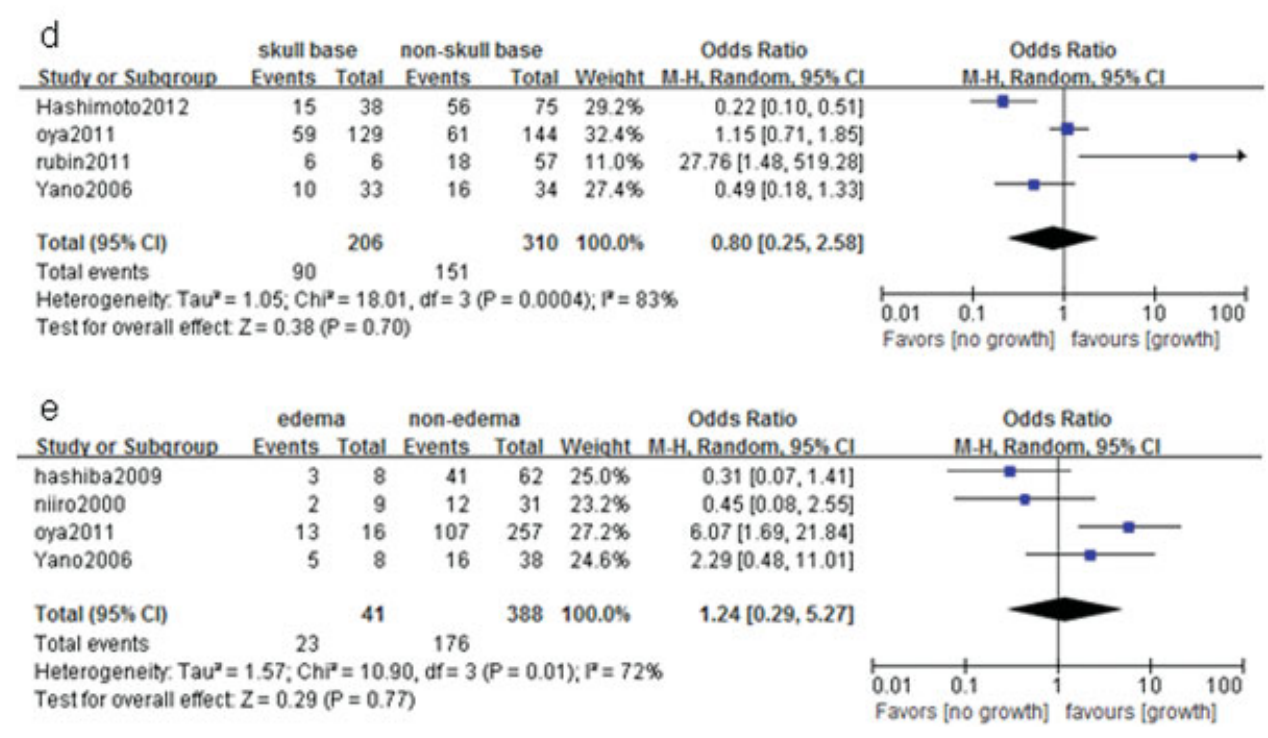

Fig. 2 Meta-analysis of the association between tumor growth on serial imaging and the clinicoradiologic factors of (A) calcification, (B) magnetic resonance imaging T2 signal intensity, (C) sex, (D) tumor location, and (E) peritumoral brain edema.

prognosis. ${ }^{11}$ Does gender affect meningioma growth? One previous study found that the growth potential of meningioma cells of male patients was significantly higher than that of females. ${ }^{12}$ However, Nakasu et al ${ }^{13}$ and Hashiba et al ${ }^{14,15}$ found no correlation between gender and tumor cell proliferation, which is consistent with the results of our meta-analysis.

Skull base meningioma accounts for a considerable proportion of asymptomatic incidental meningiomas. ${ }^{8}$ 
Table 2 Meta-analysis of factors related to tumor growth in subgroups classified according to different growth measurements on serial imaging

\begin{tabular}{|c|c|c|}
\hline & Volumetric analysis & Maximum diameter \\
\hline \multicolumn{3}{|l|}{ Male/female } \\
\hline No. of studies & $3^{15,18,26}$ & $4^{9,10,18,30}$ \\
\hline OR & 2.08 & 1.12 \\
\hline $95 \% \mathrm{Cl}$ & $0.86-5.04$ & $0.68-1.83$ \\
\hline$P$ & 0.107 & 0.662 \\
\hline $1^{2}$ & $0.0 \%$ & $0.0 \%$ \\
\hline \multicolumn{3}{|c|}{ Skull base/non-skull base } \\
\hline No. of studies & $1^{8}$ & $3^{4,9,10}$ \\
\hline OR & 0.22 & 1.24 \\
\hline $95 \% \mathrm{Cl}$ & $0.10-0.51$ & $0.33-4.64$ \\
\hline$P$ & $<0.001$ & 0.753 \\
\hline $\mathrm{I}^{2}$ & & $75.5 \%$ \\
\hline \multicolumn{3}{|c|}{ Calcification/noncalcification } \\
\hline No. of studies & $2^{15,18}$ & $4^{4,9,10,19}$ \\
\hline OR & 0.23 & 0.22 \\
\hline $95 \% \mathrm{Cl}$ & $0.10-0.53$ & $0.08-0.59$ \\
\hline$P$ & 0.001 & 0.002 \\
\hline $1^{2}$ & $42.5 \%$ & $67.4 \%$ \\
\hline \multicolumn{3}{|c|}{ MRI T2 hypersignal/hyposignal } \\
\hline No. of studies & $2^{15,18}$ & $3^{4,10,19}$ \\
\hline OR & 2.54 & 2.85 \\
\hline $95 \% \mathrm{Cl}$ & $1.12-5.76$ & $1.66-4.92$ \\
\hline$P$ & 0.026 & $<0.001$ \\
\hline $1^{2}$ & $80.7 \%$ & $0.0 \%$ \\
\hline \multicolumn{3}{|c|}{ Edema/Nonedema } \\
\hline No. of studies & $1^{15}$ & $3^{4,10,19}$ \\
\hline OR & 0.31 & 2.019 \\
\hline $95 \% \mathrm{Cl}$ & $0.07-1.41$ & $0.47-8.76$ \\
\hline$P$ & 0.129 & 0.348 \\
\hline $1^{2}$ & & $64.3 \%$ \\
\hline
\end{tabular}

Abbreviations: $\mathrm{Cl}$, confidence interval; MRI, magnetic resonance imaging; OR, odds ratio.

Because its deep location is adjacent to many important nerves and blood vessels, the operation is quite challenging and postsurgical complication rates are relatively high. ${ }^{16}$ Accordingly, a decision to operate on an asymptomatic meningioma in the skull base should be made with caution. The correlation between tumor growth and skull base location remains controversial. Hashimoto et $\mathrm{al}^{8}$ found a negative correlation of tumor growth with skull base location, whereas Rubin et $\mathrm{al}^{9}$ found a positive correlation. No correlations between tumor growth and skull base location were demonstrated by Oya et $\mathrm{al}^{10}$ and Yano and Kuratsu. ${ }^{4}$ Our results show that skull base location does not correlate with tumor growth rate. This suggests that conservative treatment of a skull base tumor should be considered.
One significant factor negatively associated with tumor growth in our analysis is calcification, a common feature of meningioma. ${ }^{17}$ Many previous studies also demonstrated this significant association. ${ }^{9,18,19}$ For instance, Nirro et al followed up meningioma patients for an average of 38.4 months using serial imaging. ${ }^{18}$ Overall, 16 of 19 cases of calcified meningiomas showed no growth; 11 of 21 noncalcified meningiomas grew significantly. Rubin et $\mathrm{al}^{9}$ followed 33 patients with calcified meningiomas for a mean period of 65 months and observed growth in only 3 cases. Eighteen of 27 noncalcified meningiomas showed obvious growth.

The meta-analysis also indicates that MRI T2 signal intensity, which mirrors tumor texture, is significantly related to meningioma growth. Low T2 signal indicates a slow growth of 
meningioma. The reasons for the low T2 signal include the following: (1) There is less water within tumor cells, (2) there are more fibrosis components, and (3) the texture of tumors is hard, such as calcification. ${ }^{20,21}$ Therefore the prognostic significance of MRI T2 signal intensity is consistent with tumor calcification.

Peritumoral brain edema frequently accompanies meningioma and may indicate an invasive growth pattern as shown on MRI that the brain-tumor interface disappears. ${ }^{22-24} \mathrm{How}$ ever, in our analysis it was not a significant factor indicating tumor growth.

Do the methods of tumor size measurements influence the results? In the included studies, two methods were used to measure tumor growth: maximum tumor diameter measurement or tumor volumetry. Maximum tumor diameter is defined by the maximum linear diameter in any direction of the tumor. At least two views of axial, coronal, and sagittal planes should be used to check the value. ${ }^{10}$ In tumor volumetry, image-analyzing software was used to trace the contour of the tumor in each slice image. The tumor volume was calculated by multiplying each tumor area by the slice thickness of the image. ${ }^{10}$ It was demonstrated that both methods can effectively detect tumor growth. The volume method is relatively more sensitive than the maximum diameter method, especially for larger tumors or tumors located in the skull base. ${ }^{25}$ Due to the different measurements for tumor growth, different studies may conclude distinct growth-related risk factors, thus leading to data heterogeneity of this study. Therefore, the subgroup analysis based on the respective growth measurement was performed again. However, the later results still confirmed tumor calcification and MRI T2 low signal as significant factors for slow tumor growth.

Our results can contribute to an optimal treatment choice for asymptomatic meningioma. For instance, if a patient has tumor calcification or a low signal in the MRI T2 sequence, a follow-up observation with neuroimaging and clinical monitoring can be preferentially considered. The follow-up interval can be as long as 6 months or 1 year. ${ }^{4}$ However, if the imaging examination does not find calcification or low signal in the MRI T2 sequence, the possibility of rapid growth exists within such tumors. However, because the patient manifests no symptoms, follow-up imaging could still be suggested at the early stage, and the follow-up interval should be shorter. As suggested by several studies, the reexamination of imaging should be done 3 months after diagnosis to exclude atypical or malignant meningioma or other nonmeningioma lesions. ${ }^{2,4,26,27}$ If the tumor still shows no obvious development, the follow-up period can be extended to 6 months or 1 year; the rate of tumor growth should be calculated every time. $2,4,26$ In cases where the tumor grows at an annual rate $>1 \mathrm{~cm}^{3}$ or tumor-related symptoms occurs, surgery needs to be considered. ${ }^{26,28-30}$ Otherwise the follow-up observation can be continued every year. ${ }^{2,4,26}$

This study has some limitations. First, in the growth assessment with serial imaging, all meningiomas were diagnosed by image rather than by pathology. Some lesions, such as hemangiopericytoma or meningeal metastasis, can mimic typical meningioma on MRI, but the first two exhibit aggressive growth behavior. ${ }^{2}$ Mixing of these nonmeningioma lesions in the meningioma group may lead to different factors related to tumor growth. Second, the included subsidiary studies did not adopt a unified criterion for definition of growth. For instance, Yoneoka et $\mathrm{al}^{26}$ defined rapid tumor growth by an annual growth $>1 \mathrm{~cm}^{3}$, whereas the criterion of Hashiba et al for rapid growth was an annual volume growth rate $>15 \%{ }^{15}$ Because each criterion of the respective study was made on its own standards to eliminate measurement bias, these diverse growth definitions were relatively comparable. ${ }^{15}$ Third, the lengths of the follow-up periods in different studies were not totally identical. Finally, in the process of the literature retrieval, only nine articles matched the full inclusion criteria. Some studies were regretfully not included because no data with a format fit for meta-analysis were presented. Therefore, we hope that future studies adopt a unified definition of tumor growth and a consistent followup duration and that they provide adequate original data. Thus the meta-analysis may maximally reduce research heterogeneity and bias.

In conclusion, we have shown that two factors, tumor calcification and MRI T2 low signal, indicate slow growth or even no growth of meningioma. No significant correlation between gender, tumor site, peritumoral edema, and tumor growth was found in our study. The results are helpful to predict the growth pattern of asymptomatic meningioma and thus choose an optimal treatment for patients.

\section{References}

1 Whittle IR, Smith C, Navoo P, Collie D. Meningiomas. Lancet 2004; 363(9420):1535-1543

2 Chamoun R, Krisht KM, Couldwell WT. Incidental meningiomas. Neurosurg Focus 2011;31(6):E19

3 Go RS, Taylor BV, Kimmel DW. The natural history of asymptomatic meningiomas in Olmsted County, Minnesota. Neurology 1998; 51(6):1718-1720

4 Yano S, Kuratsu J; Kumamoto Brain Tumor Research Group. Indications for surgery in patients with asymptomatic meningiomas based on an extensive experience. J Neurosurg 2006;105(4): 538-543

5 Firsching RP, Fischer A, Peters R, Thun F, Klug N. Growth rate of incidental meningiomas. J Neurosurg 1990;73(4):545-547

6 Braunstein JB, Vick NA. Meningiomas: the decision not to operate. Neurology 1997;48(5):1459-1462

7 Staneczek W, Jänisch W. Epidemiologic data on meningiomas in East Germany 1961-1986: incidence, localization, age and sex distribution. Clin Neuropathol 1992;11(3):135-141

8 Hashimoto N, Rabo CS, Okita Y, et al. Slower growth of skull base meningiomas compared with non-skull base meningiomas based on volumetric and biological studies. J Neurosurg 2012;116(3): 574-580

9 Rubin G, Herscovici Z, Laviv Y, Jackson S, Rappaport ZH. Outcome of untreated meningiomas. Isr Med Assoc J 2011;13(3): $157-160$

10 Oya S, Kim SH, Sade B, Lee JH. The natural history of intracranial meningiomas. J Neurosurg 2011;114(5):1250-1256

11 Pravdenkova S, Al-Mefty O, Sawyer J, Husain M. Progesterone and estrogen receptors: opposing prognostic indicators in meningiomas. J Neurosurg 2006;105(2):163-173 
12 Kasuya H, Kubo O, Tanaka M, Amano K, Kato K, Hori T. Clinical and radiological features related to the growth potential of meningioma. Neurosurg Rev 2006;29(4):293-296; discussion 296-297

13 Nakasu S, Nakajima M, Matsumura K, Nakasu Y, Handa J. Meningioma: proliferating potential and clinicoradiological features. Neurosurgery 1995;37(6):1049-1055

14 Hashiba T, Hashimoto N, Maruno M, et al. Scoring radiologic characteristics to predict proliferative potential in meningiomas. Brain Tumor Pathol 2006;23(1):49-54

15 Hashiba T, Hashimoto N, Izumoto S, et al. Serial volumetric assessment of the natural history and growth pattern of incidentally discovered meningiomas. J Neurosurg 2009;110(4):675-684

16 Di Maio S, Ramanathan D, Garcia-Lopez R, et al. Evolution and future of skull base surgery: the paradigm of skull base meningiomas. World Neurosurg 2012;78(3-4):260-275

17 Kizana E, Lee R, Young N, Dorsch NW, Soo YS. A review of the radiological features of intracranial meningiomas. Australas Radiol 1996;40(4):454-462

18 Niiro M, Yatsushiro K, Nakamura K, Kawahara Y, Kuratsu J. Natural history of elderly patients with asymptomatic meningiomas. J Neurol Neurosurg Psychiatry 2000;68(1):25-28

19 Kuratsu J, Kochi M, Ushio Y. Incidence and clinical features of asymptomatic meningiomas. J Neurosurg 2000;92(5):766-770

20 Sitthinamsuwan B, Khampalikit I, Nunta-aree S, Srirabheebhat P, Witthiwej T, Nitising A. Predictors of meningioma consistency: a study in 243 consecutive cases. Acta Neurochir (Wien) 2012; 154(8):1383-1389

21 Tenner MS, Spiller M, Koenig SH, et al. Calcification can shorten T2, but not $\mathrm{T} 1$, at magnetic resonance imaging fields. Results of a relaxometry study of calcified human meningiomas. Invest Radiol 1995;30(6):345-353

22 Lobato RD, Alday R, Gómez PA, et al. Brain oedema in patients with intracranial meningioma. Correlation between clinical, radiologi$\mathrm{cal}$, and histological factors and the presence and intensity of oedema. Acta Neurochir (Wien) 1996;138(5):485-493; discussion 493-494

23 Vignes JR, Sesay M, Rezajooi K, Gimbert E, Liguoro D. Peritumoral edema and prognosis in intracranial meningioma surgery. J Clin Neurosci 2008;15(7):764-768

24 Nakano T, Asano K, Miura H, Itoh S, Suzuki S. Meningiomas with brain edema: radiological characteristics on MRI and review of the literature. Clin Imaging 2002;26(4):243-249

25 Oya S, Sade B, Lee JH. Benefits and limitations of diameter measurement in the conservative management of meningiomas. Surg Neurol Int 2011;2:158

26 Yoneoka Y, Fujii Y, Tanaka R. Growth of incidental meningiomas. Acta Neurochir (Wien) 2000;142(5):507-511

27 Couldwell WT. Asymptomatic meningiomas. J Neurosurg 2006; 105(4):536-537; discussion 537

28 Nakamura M, Roser F, Michel J, Jacobs C, Samii M. The natural history of incidental meningiomas. Neurosurgery 2003;53(1): 62-70; discussion 70-71

29 Olivero WC, Lister JR, Elwood PW. The natural history and growth rate of asymptomatic meningiomas: a review of 60 patients. J Neurosurg 1995;83(2):222-224

30 Herscovici Z, Rappaport Z, Sulkes J, Danaila L, Rubin G. Natural history of conservatively treated meningiomas. Neurology 2004; 63(6):1133-1134 